\title{
The Multiple Mediating Effects of Perceived Social Support and Spiritual Well-being on the Relationship Between Spiritual Needs and Quality of Life Among Patients With Advanced Cancer
}

\section{Quying Wen}

Huizhou Municipal Central Hospital

Miaorui Jiao

The Affiliated Tumor Hospital of Zhengzhou University

Huailin Wang

The First Affiliated Hospital of Jilin University

Yanli Hu ( $\sim$ huyanli1212@126.com )

Ji'nan University

\section{Research Article}

Keywords: Cancer, spiritual needs, perceived social support, spiritual well-being, quality of life

Posted Date: October 18th, 2021

DOl: https://doi.org/10.21203/rs.3.rs-948082/v1

License: (c) (1) This work is licensed under a Creative Commons Attribution 4.0 International License.

Read Full License 


\section{Abstract}

Aims: The purpose of this study was to test the associations between spiritual needs, perceived social support, spiritual well-being, and quality of life (QoL) and examine the multiple mediating effects of perceived social support and spiritual well-being on the relationship between spiritual needs and QoL among patients with advanced cancer.

Methods: Spiritual needs, perceived social support, spiritual well-being and QoL were assessed using selfreport questionnaires among 286 cancer patients in a tertiary oncology hospital. The mediation model was analysed using the SPSS PROCESS procedure, and indirect effects were evaluated with bootstrapping.

Results: QoL was positively correlated with spiritual needs ( $r=0.315, p<0.01)$, perceived social support $(r=0.451, p<0.01)$ and spiritual well-being $(r=0.636, p<0.01)$. The relationship between spiritual needs and QoL was mediated by perceived social support (indirect effect: 0.063 , confidence interval $(\mathrm{Cl})(0.018$, $0.118)$ ) and spiritual well-being (indirect effect: $0.068, \mathrm{Cl}(0.001,0.155)$ ) and serially mediated by spiritual needs and QoL (indirect effect: $0.072, \mathrm{Cl}(0.041,0.113)$ ).

Conclusions: The results emphasize the importance of spiritual well-being and social support for cancer patients, especially those with more spiritual needs. They suggest that healthcare providers should develop strategies to enhance perceived social support and spiritual well-being when caring for cancer patients to improve patients' QoL.

\section{Introduction}

A progressive cancer diagnosis and the subsequent treatments such as surgery, chemotherapy, and radiation therapy treatment often create a crisis for patients[1-4], as they are confronted with deteriorating physical conditions, impaired functional ability, emotional anguish, psychological distress, and the threat of death[1-4, 5, 6]. All these adverse effects lead to a serious decline in the quality of life (QoL) of patients, which is a serious burden for individuals, families, and society[7]. As cancer generates increasing attention worldwide, effective interventions to help patients maintain good QoL become very necessary. QoL is a multidimensional concept, and measures of QoL usually include well-being, which reflects a person's positive or negative perceptions of physical, functional, emotional, psychological, social, and spiritual well-being[8-10]. Particularly for cancer patients, QoL is commonly considered a health indicator that assists in evaluating the impacts of diseases and the effects of treatments on an individual's well-being $[8,9]$. Whereas many cancer patients suffer from various stressors that could impair their QoL, meeting patients' spiritual needs has been found to be significantly associated with self-perceived good health[11, 12].

Spiritual needs are common in cancer patients[13] and have been demonstrated to be correlated with cancer patients' QoL[14, 15]. Unmet spiritual needs increase the physical and psychological symptoms of patients and reduce the effect of treatment and rehabilitation, leading to adverse prognoses such as 
depressive symptoms[15]. In addition, failure to meet spiritual needs is associated with impaired physical, emotional, psychological, social, and spiritual well-being in cancer patients[14]. A literature review suggested that the spiritual needs of cancer patients increase as a disease progresses[16] and that most patients report less spiritual care provided by health care providers than desired or even an unavailability of spiritual care[14,15,17]. Therefore, providing spiritual care to meet spiritual needs in this population is crucial. To meet spiritual needs and improve QoL, it is important to identify the mechanism of the relationship between these two variables and the variables that can be improved in cancer patients.

Cognitive appraisal theory (CAT), developed by Lazarus and Folkman[18-21], provides a framework for understanding and interpreting the role of individual cognitive behaviours in the relationship between stress and health outcomes. CAT underscores the importance of cognitive appraisal in the process of stress response and suggests that the process of action, that is, the role of cognition in the individual's stress response, finally determines the process of the stress response through many intermediary factors $[18,20]$. Folkman et al. $[18,19]$ noted that in a certain situation, the result of stress events is the decision based on the individual's cognitive appraisal process and coping process. According to the model, people generate cognitive appraisals based on their experiences of illness and treatment and the special needs during the disease period that they respond to, including factors such as perceived social support from family members, friends, and important others; social role or family role changes; and spirituality. Numerous studies have demonstrated the positive effects of cognitive behavioural therapy on improving the QoL of cancer patients[22-24]. As a cognitive appraisal factor, perceived social support directly influences health outcomes (e.g., depression symptoms, fatigue, sleep behaviour, QoL)[25-29], but it may also affect patients' spiritual well-being[30]. In the present study, spiritual needs were operationalized as a stress response of individuals threatened by cancer and QoL was operationalized as a health outcome to test the multiple mediating role of perceived social support and spiritual well-being in the relationship between spiritual needs and QoL. Figure 1 shows the proposed mediation model.

Cognitive appraisal is fundamental to CAT $[18,19]$, which proposes that "stressful events are personenvironment transactions that are appraised by the person as relevant to well-being"[19]. The outcome of a stress event depends to a certain extent on the individual's cognitive appraisal factors because the individual will use the appraisal as an avoidance mechanism or as a motivation to engage in healthy behaviour. Perceived social support has three components: support from family, friends, and important others[31,32]. Studies have shown that perceived social support plays an important role in illness outcomes[33, 34] (e.g., QoL) among cancer patients. For example, patients who perceived more support from family, friends, and important others reported higher levels of QoL[35]. Perceived social support may be one pathway through which spiritual needs affect QoL. Thus, patients' perceived social support should be addressed as a modifiable component in the development of interventions for QoL among cancer patients. 
Spiritual well-being is a complex, subjective, individualized, and latent construct[36, 37]. Although spiritual well-being lacks a unified definition across cultures, most people acknowledge that spiritual well-being is often assessed to evaluate one's spiritual experiences and outcomes[38]. Spiritual well-being can be conceptually defined in the context of disease-related health as a patient's perceptions, experiences, and feelings of well-being and health resulting from seeking intrinsic congruence; connections to nature, the sacred, and other individuals; presence in the moment; and meaning and purpose in life[39-41], as well as gaining the self-confidence to overcome existential challenges[42] and achieve the life goals of life that he or she truly wants to achieve. This operational definition has the potential to allow oncology nurses and doctors, psychologists, and healthcare managers to promote cancer patients' spiritual well-being with cognitive or/and behavioural changes via effective spiritual care interventions or educational programmes[43]. Peterman et al.[44] described two components of spiritual well-being in illness: (1) a sense of meaning and peace and (2) faith. An increasing number of studies have indicated that spiritual well-being is a significant predictor or indicator of one's perceived QoL[45-47]. Studies have reported associations between spiritual well-being and QoL among various cancer patient populations, such as breast, lung, or prostate cancer patients[38]. Empirical and theoretical work has also supported the potential of individuals to restore, maintain, and enhance their QoL after a diagnosis with life-threatening cancer, as well as the notion that spiritual well-being is one of the determinants of patient QoL[45-47].

The literature has shown a relationship between the degree of satisfaction of spiritual needs and QoL, and studies have found that spiritual well-being may function as a coping mechanism in addressing stress responses to illness. The links of perceived social support and spiritual well-being with QoL have been tested in the cancer patient population. According to CAT, perceived social support and spiritual wellbeing may be intermediate variables and may sequentially mediate the relationship between spiritual needs and QoL. However, literature on the mechanisms by which perceived social support and spiritual well-being play a serial mediating role in the relationship between spiritual needs and QoL in cancer patients is absent. To improve QoL among patients with cancer, especially among patients with greater spiritual needs, it is necessary to understand the modifiable elements that may mediate the relations for health carers, the patients themselves and their family caregivers. Therefore, this study proposes the following hypothesis: (a) perceived social support mediates the relationship between spiritual needs and QoL; (b) spiritual well-being mediates the relationship between spiritual needs and QoL; (c) perceived social support and spiritual well-being sequentially mediate the relationship between spiritual needs and QoL in patients with cancer. If these hypotheses are confirmed, they can potentially be used to meet spiritual needs and improve QoL among cancer patients.

\section{Methods}

\subsection{Design and sample}

This study was a cross-sectional study. Participants were recruited from July 2020 to October 2019 from an oncology university hospital in China using a convenience sampling method and were asked to 
provide written informed consent. The present study was reviewed and approved by the Institutional Review Board of the Henan Province Medical Science and Technology Research Plan (Joint Construction) Project (\#LHGJ20190654). Participants completed the questionnaires, and nurses collected them on the spot. The inclusion criteria were as follows: (a) age $\geq 18$ years old, (b) a confirmed diagnosis of cancer by a physician, and (c) stage II-IV cancer according to the American Joint Committee on

Cancer (AJCC) eighth edition cancer staging manual. The exclusion criteria were as follows: patients who were extremely frail, had a mental illness, had visual or hearing impairments, or who were illiterate.

We found a moderate $f^{2}$ effect size of 0.15 , an a of 0.05 and a power of 0.90 (using G*Power Version 3.1, written by Franz Faul). We calculated a patient sample size of 206 and assumed a dropout rate of $20 \%$; thus, two hundred eighty-six participants were sufficient for the study.

\subsection{Measures}

Demographic and clinical characteristics. The demographic characteristics included gender, age, marital status, working status, educational level, and monthly income. The clinical characteristics included diagnosis, chemotherapy/radiotherapy, somatic pain score, and cancer duration, as obtained from patient self-reports or medical records.

Spiritual needs. The original 26-item Korean version of the Spiritual Needs Scale (SNS) developed by Yong[48] measures the spiritual needs of patients with cancer. It consists of five factors with Cronbach's alpha values ranging from 0.74 to 0.91 . Spiritual needs were measured in this study using the 23-item Chinese version of the SNS which was translated, cross-culturally adapted, and evaluated by Cheng[49]. It has five different components with good psychometric properties: relationship with God (divine, sacred; Cronbach's alpha=0.65); meaning and purpose (Cronbach's alpha 0.81 ); acceptance of dying (Cronbach's alpha=0.79); hope and peace (Cronbach's alpha=0.74); and love and connection (Cronbach's alpha=0.79). The Chinese version of the SNS uses a five-point Likert response scale ranging from one (not at all) to five (a great deal). Higher scores on the scale indicate more spiritual needs.

Perceived social support. The 12-item self-report Multidimensional Scale of Perceived Social Support (MSPSS) developed by Zimet et al.[50-52] assesses persons' perceived social support from family, friends, and significant others. The Chinese version of the Scale of Perceived Social Support (SPSS) includes two subscales (perceived social support from within the family and outside the family) and was translated and evaluated by Huang et al[53]. It uses a 7-point Likert scale (from very strongly disagree to very strongly agree), and higher scores indicate more perceived social support. A literature review demonstrated that the SPSS has sound internal reliability, test-retest reliability, and factorial validity.

Spiritual well-being. The 12-item Functional Assessment of Chronic Illness Therapy-Spiritual Well-Being Scale (FACIT-Sp-12) (v. 4)[54,55] uses a 5-point Likert-type scale (0区"not at all”; 4区"very much”). Higher scores reflect higher levels of spiritual well-being. The FACIT-Sp-12 comprises three subscales: sense of meaning, sense of peace, and the role of faith in illness. The Cronbach's alphas for the three subscales ranged from 0.80 to $0.93[55]$. The Chinese version of the FACIT-Sp-12 scale was translated and evaluated 
by Liu et al.[56]. The Cronbach's a coefficients of the three subscales ranged from 0.71 to 0.92 . The Chinese version of the FACIT-Sp-12 scale has shown good reliability and validity, and it can be used as an assessment tool for the QoL of Chinese cancer patients.

Quality of life. QoL was measured with the Functional Assessment of Cancer Therapy-General (FACT-G) (v.4) developed by Cella et al[57]. It has 27 items with a 5-point Likert-type scale. The FACT-G assesses four components: physical well-being, functional well-being, emotional well-being, and social/family wellbeing. The Cronbach's alphas for the four subscales ranged from 0.85 to 0.88 . It is a widely used measure of QoL. The Chinese version of the FACT-G[56] also showed sound reliability and validity.

\subsection{Statistical Analysis}

Data analysis was conducted using SPSS version 23.0 for Windows (IBM Corp, Armonk, NY, USA). A value of $p<0.05$ was considered statistically significant. We computed percentages and frequencies for the categorical variables and means and standard deviations for the continuous variables. The bivariate correlations of spiritual needs, perceived social support and spiritual well-being with QoL were examined by Pearson product-moment correlation coefficients. We determined the impact factors of QoL among cancer patients using multivariate linear regression. We performed mediation analysis to test whether the association between spiritual needs and QoL was serially mediated by perceived social support and spiritual well-being. It was assumed that spiritual needs $(X)$ would be associated with perceived social support $\left(M_{1}\right)$, which, in turn, would generate spiritual well-being $\left(M_{2}\right)$ and influence QoL $(Y)$. A serial mediator model with two mediators (perceived social support and spiritual well-being) provided three indirect effects and a total indirect effect. The indirect effects included in this model were (1) perceived social support $\left(a_{1} b_{1}\right),(2)$ spiritual well-being $\left(a_{2} b_{2}\right)$, and (3) perceived social support and spiritual wellbeing $\left(\mathrm{a}_{1} \mathrm{~d}_{21} \mathrm{~b}_{2}\right)$. The SPSS PROCESS procedure developed by Preacher and Hayes[58] was used to test the proposed serial mediation model (model 6 and 5000 bootstrap samples). The direct and indirect effects were considered significant when the corrected bootstrap confidence interval ( $\mathrm{Cl})$ did not include zero.

\section{Results}

\subsection{Participant characteristics}

Among the 286 participants in this study, the mean age was $53.2 \pm 13.5$ years, $63.3 \%$ were male, and $91.3 \%$ were cohabiting or married. The top three cancer types in this sample were gastrointestinal cancer 93 (32.5\%), lung cancer 71 (24.8\%), and breast cancer 29 (10.1\%). A majority of the patients $(74.2 \%)$ experienced cancer for less than 12 months. Nearly half of the participants (47.9\%) had more than four points of somatic pain measured using a pain thermometer. In addition, $61.5 \%$ of the patients had less than a high school education, $66.8 \%$ were on duty, and $42.3 \%$ earned less than $\otimes 1000$ per month. Table 1 shows more details of the sample characteristics.

\subsection{Mean scores and correlation coefficients of the study variables}


As shown in Table 2, the mean scores for spiritual needs, perceived social support, spiritual well-being, and QoL were 82.59 $\pm 19.10,62.35 \pm 13.86,36.13 \pm 10.37$, and 69.86 \pm 16.65 , respectively. Spiritual needs were positively associated with perceived social support $(r=0.400, p<0.01)$, spiritual well-being $(r=0.302$, $p<0.01)$, and QoL $(r=0.315, p<0.01)$. Perceived social support was positively associated with spiritual wellbeing $(r=0.446, p<0.01)$ and QoL $(r=0.451, p<0.01)$. Spiritual well-being was positively associated with QoL $(r=0.636, p<0.01)$.

\subsection{Multivariable linear regression analysis of quality of life}

The results of this study revealed that all variance tolerances were higher than 0.5 , and all variance inflation factors were lower than 2, suggesting that there was no multicollinearity. The number of children, pain rating, spiritual needs, perceived social support and spiritual well-being were associated with QoL, accounting for $45.7 \%$ of the variance in QoL (Table 3 ).

\subsection{Multiple mediating effects of perceived social support and spiritual well-being}

The indirect effects of spiritual needs on QoL via both perceived social support and spiritual well-being were examined using serial mediation mode 6 with a 95\% Cl based on 5000 bootstrapping samples[59]. All the mediated indirect effects of each specific path are shown in Figure 2 and Table 4. The results of this analysis indicated that the total effect of spiritual needs on QoL was significant $(c=0.275, \mathrm{Cl}(0.178$, 0.372)). Spiritual needs had a significant indirect effect on QoL through perceivedsocial support and then spiritual well-being (a1d21b2=0.072, $\mathrm{Cl}(0.041,0.113)$, which accounted for $26.02 \%$ of the total effect of spiritual needs on QoL. In addition, spiritual needs had significant indirect effects on QoL via both perceived social support $(\mathrm{a} 1 \mathrm{~b} 1=0.063, \mathrm{Cl}(0.018,0.0118)$ ) and spiritual well-being $(\mathrm{a} 2 \mathrm{~b} 2=0.068, \mathrm{Cl}(0.001$, 0.155)), accounting for $23.00 \%$ and $24.79 \%$ of the total effect of spiritual needs on QoL, respectively.

However, we did not find a significant direct effect of spiritual needs on QoL (direct effect of spiritual needs on QoL: $\left.c^{\prime}=0.072, \mathrm{Cl}(-0.012,0.156)\right)$ in patients with cancer.

\section{Discussion}

To date, the interplay between spiritual needs, perceived social support, spiritual well-being, and QoL has not been evaluated via a mediation model. Several studies have found an association between spiritual needs and QoL; however, few studies have explored the possible mechanism for this relationship. The results of this study revealed that cancer patients with more spiritual needs perceived more social support and tended to use spiritual well-being as a coping strategy, which led to higher levels of QoL. This finding is consistent with CAT[18]. Spiritual needs had no significant direct effect on QoL. However, they had an indirect effect on QoL serially mediated by perceived social support and spiritual well-being.

The average QoL score (total scale score range: 0- to 108 points) of the cancer patients was in the low-tomoderate level $(M=69.86(S D=16.65))$, which was congruent with previous studies $[59,60]$ with cancer patients that employed the Quality of Life Questionnaire-Core 30 (QLQ -C30) in China. However, cancer 
patients showed higher levels of spiritual needs, with an average score of $82.59(S D=19.10)$ (total scale scores range: 23 to 115), which was congruent with a previous study[13] with cancer patients that employed the 26 -item English version of the SNS. Höcker's survey showed that more than $94 \%$ of patients reported at least one spiritual need, and no significant associations for medical characteristics were observed. The results emphasized the relevance of QoL and spiritual needs for cancer patients. The call for spiritual care programmes to meet spiritual needs and enhance QoL in cancer patients is strengthened.

This study demonstrated that perceived social support mediated the relationship between spiritual needs and QoL in cancer patients. Experiences of a life-threatening illness led to the patients' stress responses and triggered their internal needs and cognitive appraisals, which promoted their emotional well-being and healthy behaviour and, eventually, positive illness outcomes[18, 33, 34]. Due to the risk of cancer recurrence, the pain of treatment, and the natural threat to patients' survival, patients experienced increased spiritual needs and social support. Insufficient patient social support leads to poor perceived social support, serious psychological distress and decreased QoL[25, 28, 29]. Perceived social support has also been viewed as a significant factor in coping with physical symptoms[61], contributing to spiritual well-being[62], decreasing loneliness and mental health problems[63]. Thus, interventions should focus on the role of social support in improving QoL and the development of strategies to promote support from family and non-family members for patients with cancer.

This current study revealed that spiritual well-being mediated the relationship between spiritual needs and QoL. Thus, hypothesis 2 was supported. Additionally, spiritual well-being may be a mechanism of the relationship of disease responses and coping behaviour with healthy outcomes, consistent with Scheffold[42] and Li's[64] research. Studies have found that people turn to spirituality and desire spiritual well-being in times of crisis[13,38]. Individuals with cancer who used spiritual well-being to cope with their illness had higher levels of QoL, which was supported by previous studies[48]. Maintaining cancer treatment and living with disease was challenging, so patients who adopted spiritual coping strategies were likely to mitigate the negative emotions of their illness responses and experience positive effects of disease. Patients with spiritual coping strategies had more feelings hope, peace, meaning, and confidence, which contributed to higher levels of QoL[48]. Thus, health care providers should assess patients' spiritual well-being to promote beneficial coping strategies and thus improve QoL in patients with cancer.

We discovered a serial mediating effect of perceived social support and spiritual well-being on the relationship between spiritual needs and QoL, which supported hypothesis 3 . That is, the indirect effect of spiritual needs on QoL through perceived social support and spiritual well-being was significant even though no significant direct effect of spiritual needs on QoL was observed. According to CAT, individuals created cognitive appraisals based on their experiences and responses to illness, which helped them seek and appreciate the importance of support from inside and outside the family and guided them to use spiritual well-being to manage their diseases. Previous studies[28-30] reported direct effects of perceived social support on psychological well-being and indirect effects mediated by coping. When cancer patients 
feel that their lives are threatened, they may have strong desires for social support[65]. In addition, perceived social support was shaped directly in response to patients' illnesses and thus influenced the use of spiritual well-being to address the condition. Spiritual well-being as an adaptive coping strategy tended to encourage individuals to meet their spiritual needs and form positive cognitive appraisals about their diseases, resulting in their ability to achieve peace, meaning, life goals, self-transcendence, and rehabilitation from cancer. Therefore, healthcare professionals should evaluate cancer patients' spiritual needs, improve their perceived social support and help them develop appropriate spiritual wellbeing to improve and maintain QoL.

\subsection{Implications for clinical practice}

Although it is widely acknowledged that people have more spiritual needs in times of threatening illness, interest in exploring the spiritual needs and spiritual well-being of cancer patients and corresponding spiritual care is just beginning to grow in China. The study findings have key implications for meeting patients' spiritual needs and improving their QoL. Spiritual needs were found to be a common demand among cancer patients that could not be neglected[13]. Consequently, hospitals should create conditions that allow and encourage patients' families, relatives, colleagues, friends to visit and accompany them. Healthcare providers should assess patients' spiritual needs when evaluating their health statuses and organize various forms of health education activities that promote their spiritual well-being to improve their perceived social support (e.g., patient group activities, patient-caregiver dyadic intervention programmes, or various social/cultural activities) and spiritual well-being (e.g., spiritual care, expression of emotions, life review, life and death education), thereby improving their QoL.

Rehabilitation therapists, community nurses, physicians and staff, together with the family caregivers of cancer patients, should provide more social support (e.g., information provision and communication[66], structured psycho-oncological care model from healthcare staff[67], and encourage individuals to participate in various social activities tailored to patients' competencies and interests. Staff should take social support and spiritual well-being into account when developing health plans for cancer patients. Levels of perceived social support and spiritual well-being should be indicators for hospital quality evaluation.

\subsection{Strengths and limitations}

This is the first study to simultaneously investigate spiritual needs, perceived social support, spiritual well-being, and QoL among cancer patients. It is also provides the first evidence of the mechanism by which perceived social support and spiritual well-being exert their mediating role in the relationship between spiritual needs and QoL. This study helped explain the impact of spiritual needs on cancer patients' QoL.

Despite the above strength, some limitations must be acknowledged. First, the sample of this study was from a large oncology hospital in China recruited using a convenient sampling method. This group may

not be sufficient to represent all cancer patients in China. Second, spiritual needs, spiritual well-being, and 
QoL are situational factors that may vary during the illness trajectory. Thus, future studies should conduct longitudinal studies. Third, this survey relied on self-report methods, which may have led to bias. Moreover, the FACT-G is a multidimensional scale that includes physical, emotion, family/social, and functional domains, and the Chinese version of the MSPSS consists of two subscales, as do the SNS and FACIT-Sp. However, this study used the total scale scores of the various scales for the evaluations and did not separately assess the relationships between the various dimensions. A future study may investigate the relationship between the various components.

\section{Conclusion}

These findings revealed the theoretical relationships between spiritual needs, perceived social support, spiritual well-being and health outcomes and expanded CAT to the cancer research area. Spiritual needs, together with perceived social support and spiritual well-being, had an indirect effect on QoL. Additionally, perceived social support and spiritual well-being played a serial mediating role in the relationship between spiritual needs and QoL. Therefore, effective programmes that can improve patients' perceived social support and/or spiritual well-being may be beneficial in helping cancer patients, especially those who have more spiritual needs, improve their QoL. Future research to develop culturally based spiritual care interventions for cancer patients is suggested.

\section{Abbreviations}

QoL: quality of life; Cl: confidence interval; CAT: Cognitive appraisal theory; AJCC : American Joint Committee on Cancer; SNS: Spiritual Needs Scale; MSPSS: Multidimensional Scale of Perceived Social Support; SPSS: Scale of Perceived Social Support; FACIT-Sp: Functional Assessment of Chronic Illness Therapy-Spiritual Well-Being Scale; FACT-G: Functional Assessment of Cancer Therapy-General.

\section{Declarations}

\section{Ethics approval and consent to participate}

Informed consent was obtained from all participants (the consent obtained was written). The present study have been performed in accordance with the Declaration of Helsinki and has been reviewed and approved by the Institutional ethics committee

of the Tumor Hospital of Henan Province, China (access number: 2019014)『and the methods of the current research were carried out in accordance with the relevant guidelines and regulations involving human participants of the above institutional review board $\square$

\section{Consent for publication}

Not applicable. 
Availability of data and material

The datasets generated and/or analyzed in the current study are available upon request from the coauthor Yanli Hu in the format of SPSS files.

\section{Competing interests}

The authors declare that they have no competing interests.

\section{Funding}

This work was supported by grants from the Henan Province Medical Science and Technology Research Plan (Joint Construction) Project (\#LHGJ20190654). The funding agencies did not have any role in the design of the study, the collection, analysis, and interpretation of the data, or the writing of the manuscript.

\section{Authors' contributions}

Wen and Jiao wrote the article, Wang made the figure and table of the article, Hu wrote and revised the article.

WQY区JMR,WHL and HYL made substantial contributions to the conception, design, acquisition of the data, or analysis and interpretation of the data; WQY and JMR were involved in drafting or critically revising the manuscript for important intellectual content; WQY, JMR,WHL and HYL gave final approval of the version to be published. Each author participated sufficiently in the work to take public responsibility for the appropriate portions of the content; WQY and HYL agree to be accountable for all aspects of the work by ensuring that questions related to the accuracy or integrity of any part of the work are appropriately investigated and resolved.

\section{Acknowledgements}

We gratefully thank all participants and the oncology nurses Yizhuo, Fangfang, and Xiuli and my sister, Xiaoqi, who voluntarily participated in the survey.

\section{References}

1. Goswami S, Peipert BJ, Mongelli MN, et al. Clinical factors associated with worse quality-of-life scores in United States thyroid cancer survivors[J]. Surgery, 2019:69-74.

2. Matsuoka H, Nakamura K, Matsubara $Y$, et al. The influence of chemotherapy-induced peripheral neuropathy on quality of life of gynecologic cancer survivors[J]. International Journal of Gynecological Cancer, 2018, 28(7):1394-1402.

3. Pisu M, Azuero A, Halilova Kl, et al. Most impactful factors on the health-related quality of life of a geriatric population with cancer[J]. Cancer, 2017, 124(3), 596-605. 
4. Heins MJ. Verloop KD. Ligt S. et al. Primary Secondary Cancer Care Registry (PSCCR): Following breast cancer patients from their first complaints up to 15 years after diagnosis. European Journal of Cancer, 2018, 92, S39-S40.

5. Marco D, White M. The impact of cancer type, treatment, and distress on health-related quality of life: cross-sectional findings from a study of Australian cancer patients[J]. Supportive Care in Cancer, 2019,1-9.

6. Goncharova M, Grey J, Druce M. Impact of gastrointestinal symptoms on quality of life in MEN2. Clin Endocrinol (Oxf). 2021 Apr;94(4):606-615.

7. Ramasubbu SK, Pasricha RK, Nath UK, et al. Quality of life and factors affecting it in adult cancer patients undergoing cancer chemotherapy in a tertiary care hospital. Cancer Rep (Hoboken). 2021 Apr;4(2):e1312.

8. Aronson NK. The European Organization for Research and Treatment of Cancer QLQ-C30: a qualityof-life instrument for use in international clinical trials in oncology[J]. J. Natl. Cancer. Inst. $1993,85$.

9. Winstead-Fry P, Schultz A. Psychometric analysis of the functional assessment of cancer therapygeneral (FACT-G) scale in a rural sample[J]. Cancer, 1997, 79(12):2446-2452.

10. Brucker PS, Yost K, Cashy J, et al. General population and cancer patient norms for the Functional Assessment of Cancer Therapy-General (FACT-G)[J]. Evaluation \& the Health Professions, 2005, 28(2):192-211.

11. Cheng Q, Xu X, Liu X, et al. Spiritual needs and their associated factors among cancer patients in China: a cross-sectional study[J]. Supportive Care in Cancer, 2018, 26(10):3405-3412.

12. Moosavi S, Rohani C, Borhani F, et al. Spiritual care experiences by cancer patients, their family caregivers and healthcare team members in oncology practice settings: A qualitative study. Explore (NY). 2021 Sep-Oct;17(5):430-437.

13. Palmer Kelly E, Hyer JM, Paredes AZ, et al. Provision of supportive spiritual care for hepatopancreatic cancer patients: an unmet need? HPB (Oxford). 2021 Feb 7:S1365-182X(21)00035-6.

14. Finucane A, Swenson C, Macartney JI, et al. What Makes Palliative Care Needs "Complex"?: A Multisite Sequential Explanatory Mixed Methods Study of Patients Referred to Specialist Palliative Care[J]. BMC Palliat Care, 2021. 20(1):18.

15. Pearce MJ, Coan AD, li JEH, et al. Unmet spiritual care needs impact emotional and spiritual wellbeing in advanced cancer patients[J]. Supportive Care in Cancer, 2012, 20(10):2269-2276.

16. Murray SA, Kendall M, Grant E, et al. Patterns of social, psychological, and spiritual decline toward the end of life in lung cancer and heart failure.[J]. Journal of Pain \& Symptom Management, 2007, 34(4):393-402.

17. Selman LE, Brighton LJ, Sinclair S, et al. Patients' and caregivers' needs, experiences, preferences and research priorities in spiritual care: A focus group study across nine countries[J]. Palliative Medicine, 2017, 32(1):216-230.

18. Folkman S. Dynamics of a stressful encounter: Cognitive appraisal, coping, and encounter outcomes[J]. Journal of Personality and Social Psychology, 1986, 50(5):992-1003. 
19. Folkman S, Chesney M, Mckusick L, et al. Translating Coping Theory into an Intervention[M]// The Social Context of Coping. 1991.

20. Folkman S, Lazarus RS, Pimley S, et al. Age differences in stress and coping processes.[J]. Psychology and Aging, 1987, 2(2):171-184.

21. Moors A. Appraisal Theory of Emotion[M]. 2017.

22. Chambers SK, Ritterband LM, Thorndike F, et al. Web-Delivered Cognitive Behavioral Therapy for Distressed Cancer Patients: Randomized Controlled Trial:[J]. Journal of Medical Internet Research, 2018, 20(1):e42.

23. Hjg A, Mfm G, Rrt D, et al. The efficacy of Internet-based cognitive behavioral therapy for severely fatigued survivors of breast cancer compared with care as usual: A randomized controlled trial[J]. Cancer, 2017, 123(19):3825-3834.

24. Zhang Q, Li F, Zhang H, et al. Effects of nurse-led home-based exercise \& cognitive behavioral therapy on reducing cancer-related fatigue in patients with ovarian cancer during and after chemotherapy: A randomized controlled trial[J]. International Journal of Nursing Studies, 2017, 78:52-60.

25. Jeong A, Shin DW, Kim SY, et al. Avoidance of cancer communication, perceived social support, and anxiety and depression among patients with cancer[J]. Psycho-Oncology, 2016, 25(11):1301-1307.

26. Yoo H, Shin DW, Jeong A, et al. Perceived social support and its impact on depression and healthrelated quality of life: a comparison between cancer patients and general population[J]. Japanese Journal of Clinical Oncology, 2017, 47(8):728-734.

27. Kadambi S, Soto-Perez-de-Celis E, Garg T, et al. Social support for older adults with cancer: Young International Society of Geriatric Oncology review paper. J Geriatr Oncol. 2020, 11(2):217-224.

28. Eom CS, Shin DW, Kim SY, et al. Impact of perceived social support on the mental health and healthrelated quality of life in cancer patients: results from a nationwide, multicenter survey in South Korea[J]. Psycho-Oncology, 2013, 22(6):1283-1290.

29. Haviland J, Sodergren S, Calman L, et al. Social support following diagnosis and treatment for colorectal cancer and associations with health-related quality of life: Results from the UK ColoREctal Wellbeing (CREW) cohort study[J]. Psycho-Oncology, 2017, 26(12): 2276-2284.

30. Jun WH, Cha KS, Lee KL. The mediating effect of depression on the relationship between social support, spirituality and burnout in family members of patients with cancer. Int $\mathrm{J}$ Environ Res Public Health. 2021, 18(4):1727.

31. Zhou K, Li H, Wei X, et al. Reliability and validity of the multidimensional scale of perceived social support in Chinese mainland patients with methadone maintenance treatment[J]. Comprehensive Psychiatry, 2015, 60:182-188.

32. Hardan-Khalil K, Mayo AM. Psychometric Properties of the Multidimensional Scale of Perceived Social Support[J]. Clinical Nurse Specialist, 2017, 25(4):1885-1895.

33. Levy SM, Herberman RB, Whiteside T, et al. Perceived social support and tumor estrogen/progesterone receptor status as predictors of natural killer cell activity in breast cancer 
patients.[J]. Psychosomatic Medicine, 1990, 52(1):73-85.

34. Abdallah A, Mark L. Psychosocial-spiritual interventions among Muslims undergoing treatment for cancer: an integrative review [J]. BMC Palliat Care, 2021. 20(1):51.

35. Trevino KM, Fasciano K, Block S, et al. Correlates of social support in young adults with advanced cancer[J]. Supportive Care in Cancer, 2013, 21(2):421-429..

36. Ellison CW. Spiritual Well Being: Conceptualization and Measurement[J]. Journal of Psychology \& Theology, 1983, 11(4):330-340.

37. Munoz AR, Salsman JM, Stein KD, et al. Reference values of the Functional Assessment of Chronic Illness Therapy-Spiritual Well-Being: A report from the American Cancer Society $\backslash$ "s studies of cancer survivors[J]. Cancer, 2015, 121(11):1838-1844.

38. Chen J, Lin Y, Yan J, Wu Y, et al. The effects of spiritual care on quality of life and spiritual well-being among patients with terminal illness: A systematic review. Palliative Medicine, 2018, 32(7), 11671179.

39. Koenig HG, McCullough ME, Larson DE: Handbook of Religion and Health. Oxford, England: Oxford University Press, 2001.

40. Zinnbauer BJ, Pargament KI, Scott AB: The emerging meanings of religiousness and spirituality: Problems and prospects. Journal of Personality. 1999, 67: 889-919.

41. Ghorbani M, Mohammadi E, Aghabozorgi R,et al. Spiritual care interventions in nursing: an integrative literature review. Support Care Cancer. 2021, 29(3):1165-1181.

42. Scheffold K, Philipp R, Vehling S, et al. Spiritual well-being mediates the association between attachment insecurity and psychological distress in advanced cancer patients[J]. Supportive Care in Cancer, 2019, 27(11): 4317-4325.

43. Lee YH, Salman A. Evaluation of using the Chinese version of the Spirituality Index of Well-Being (SIWB) scale in Taiwanese elders.[J]. Applied Nursing Research, 2016, 32:206-211.

44. Peterman AH, Fitchett G, Brady MJ, et al. Measuring spiritual well-being in people with cancer: the functional assessment of chronic illness therapy-Spiritual Well-being Scale (FACIT-Sp).[J]. Annals of Behavioral Medicine, 2002, 24(1):49-58.

45. Zimmermann C, Swami N, Krzyzanowska M, et al. Early palliative care for patients with advanced cancer: a cluster randomized controlled trial. Lancet, 2014, 383(9930):1721-30.

46. Asgeirsdottir GH, Sigurdardottir V, Gunnarsdottir S, et al. Spiritual well-being and quality of life among Icelanders receiving palliative care: data from Icelandic pilot-testing of a provisional measure of spiritual well-being from the European Organisation for Research and Treatment of Cancer. Eur $\mathrm{J}$ Cancer Care (Engl), 2017, 26(2):12394.

47. Hu YanLi, Liu Tao, Li Fan. Association between dyadic interventions and outcomes in cancer patients: a meta-analysis[J]. Supportive Care in Cancer. 2019, 27(3):745-761.

48. Yong J, Kim J, Han SR, et al. Development and Validation of a Scale Assessing Spiritual Needs for Korean Patients with Cancer. Journal of Palliative Care, 2008, 24(4): 240-246. 
49. Cheng Q, Liu X, Yan Y, et al. Study on the reliability and validity of the Chinese version of the spiritual demand scale [J]. Journal of Nursing Science, 2018, 33(3):16-19.

50. Zimet T, DAHLGM NW, ZWT SG, et al. The Multidimensional Scale of Perceived Social Support. Journal of Personality Assessment, 1988, 52, 30-41.

51. Zimet GD, POWELL S, FARLEY GK, et al. Psychometric characteristics of the Multidimensional Scale of Perceived Social Support. Journal of Personality Assessment, 1990, 55, 610-617.

52. Dahlem N W, Zimet G D, Walker R R. The Multidimensional Scale of Perceived Social Support: A confirmation study[J]. J Clin Psychol, 1991, 47(6):756-761.

53. Huang L, Jiang G, Ren W. Correlation between Coping Style, Social Support and Psychosomatic Symptoms of Cancer Patients[J]. Chinese Journal of Mental Health, 1996(04): 160-161.

54. Peterman AH, Fitchett G, Brady MJ, et al. Measuring spiritual well-being in people with cancer: The functional assessment of chronic illness therapy-spiritual well-being scale (FACIT-Sp)[J]. Annals of Behavioral Medicine, 2002, 24(1):49-58.

55. Murphy PE, Canada AL, Fitchett G, et al. An examination of the 3-factor model and structural invariance across racial/ethnic groups for the FACIT-Sp: a report from the American Cancer Society \"s Study of Cancer Survivors-II (SCS-II)[J]. Psychooncology, 2010, 19(3):264-272.

56. Xiangyu L, Di W, Yongyi Z. Chinese version of chronic disease treatment function assessment - the reliability and validity of the spiritual scale in cancer patients [J]. Chinese Journal of Nursing, 2016, 51 (9): 1085-1089.

57. Cella DF, Tulsky DS, Gray G, et al. The Functional Assessment of Cancer Therapy Scale: Development and validation of the general measure. Journal of Clinical Oncology, 1993, 11(3), 570-579.

58. Preacher KJ, Hayes AF. Asymptotic and resampling strategies for assessing and comparing indirect effects in multiple mediator models. Behav Res Methods 2008, 40(3):879-891.

59. Jianping $W$, Junnan $C$, Zhonggeng $C$, et al. Study on quality of life and influencing factors in cancer patients[J]. Chinese Journal of Clinical Psychology, 2000, 8(1): 23-26.

60. Fu Wei, Li Junying, Li Hong, et al. Correlation between social support and quality of life of cancer patients and nursing strategies[J]. Chinese Journal of Nursing, 2004, 39(1): 9-11.

61. Ekwunazu C, Sutton A, Hale M, et al. Perceived social support is associated with decreased heat painrelated activity in the anterior cingulate cortex[J]. Journal of Pain, 2018, 19(3):S12-S13.

62. Ciria-Suarez L, Calderon C, Fernández Montes A, et al. Optimism and social support as contributing factors to spirituality in Cancer patients. Support Care Cancer. 2021, 29(6):3367-3373.

63. Wang J, Mann F, Lloydevans B, et al. Associations between loneliness and perceived social support and outcomes of mental health problems: a systematic review[J]. Bmc Psychiatry, 2018, 18(1):156.

64. Lee Y-H, Salman, A. The Mediating Effect of Spiritual Well-being on Depressive Symptoms and Health-related Quality of Life Among Elders. Archives of Psychiatric Nursing, 2018, 32(3):418-424.

65. Korotkin BD, Hoerger M, Voorhees S, et al. Social support in cancer: How do patients want us to help? J Psychosoc Oncol. 2019, 37(6):699-712. 
66. Kadambi S, Soto-Perez-de-Celis E, Garg T, et al. Social support for older adults with cancer: Young International Society of Geriatric Oncology review paper. J Geriatr Oncol. 2020 Mar;11(2):217-224.

67. Taylor K, Singer S, May M, et al. Outcome comparison of integrated psycho-oncological care versus unstructured care-Results of a non-randomised open-label two-arm trial[J]. European Journal of Cancer Care, 2019, 28(5):e13127.

\section{Tables}

Table 1. Characteristics of samples $(N=286)$ 
Variable

n (\%)

Mean

(SD)

Age, years

53.2

\section{Gender}

Male

Female

105

(36.7)

Diagnosis

Gastrointestinal cancer

93 (32.5)

Lung cancer

$71(24.8)$

Breast cancer

29 (10.1)

Liver cancer,

$12(4.2)$

Other types of cancer (cervical cancer, bladder cancer, lymphoma, leukemia, et al.)

$81(28.3)$

Chemotherapy/radiotherapy

Yes

No

Marital status

Unmarried

Divorced / widow

Cohabiting/married

Working status

Unemployment

Retired

Vacation/retirement because of illness

On duty

Education level

$<$ High school
193(67.5)

93(32.5)

10 (3.5)

$15(5.2)$

261

(91.3)

$23(8.0)$

41 (14.3)

31 (10.8)

191

(66.8) 
Income monthly per person

$<1000$ RMB

$\varangle 3000 \mathrm{RMB}$

$\geq 3000$ RMB

51 (17.8)

Somatic pain score

$\varangle 4$ points

\7 points

$80(28.0)$

$\geq 7$ points

57 (19.9)

Cancer duration

$<12$ months

212

(74.1)

$\geq 12$ months

$74(25.9)$

SD: standard deviation

Table 2. Mean scores and correlation coefficients of variables $(N=286)$

\begin{tabular}{lllll} 
Variable & Mean (SD) & 1 & 2 & 3 \\
\hline 1 Spiritual needs & $82.59(19.10)$ & - & - & - \\
\hline 2 Perceived social support & $62.35(13.86)$ & $0.400^{\star \star}$ & - & - \\
\hline 3 Spiritual well-being & $36.13(10.37)$ & $0.302^{\star \star}$ & $0.446^{* \star}$ & - \\
\hline 4 Quality of Life & $69.86(16.65)$ & $0.315^{\star \star}$ & $0.451^{\star \star}$ & $0.636^{* \star}$
\end{tabular}

SD: standard deviation

${ }^{\star *} p<0.01$

Table 3. Multivariable regression analysis of quality of life 


\begin{tabular}{llllll} 
Variable & $\beta$ & $\mathrm{t}$ & $P$ & \multicolumn{2}{l}{ Collinearity } \\
\hline & & & & Tolerance & VIF \\
\hline Spiritual needs & 0.088 & 1.735 & 0.084 & 0.737 & 1.357 \\
\hline Perceived social support & 0.198 & 3.735 & 0.000 & 0.681 & 1.467 \\
\hline Spiritual well-being & 0.502 & 9.616 & 0.000 & 0.702 & 1.424 \\
\hline Number of children & 0.127 & 2.516 & 0.012 & 0.754 & 1.325 \\
\hline Pain rating & 0.112 & 2.376 & 0.018 & 0.855 & 1.170
\end{tabular}

VIF: variance inflation factor.

$R^{2}=0.486$, adjusted $R^{2}=0.457$

Table 4. Mediation analysis of spiritual needs and quality of life

\begin{tabular}{lllll} 
& Effect & SE & LLCl & ULCI \\
\hline Total effect & 0.275 & 0.049 & 0.178 & 0.372 \\
\hline Direct effect & 0.072 & 0.043 & -0.012 & 0.156 \\
\hline Indirect effect & & & & \\
\hline Total & 0.203 & 0.053 & 0.102 & 0.307 \\
\hline Ind1 & 0.063 & 0.026 & 0.018 & 0.118 \\
\hline Ind2 & 0.072 & 0.018 & 0.041 & 0.113 \\
\hline Ind3 & 0.068 & 0.040 & 0.001 & 0.155
\end{tabular}

Indirect effect key:

Ind1: spiritual needs $\rightarrow$ Perceived social support $\rightarrow$ quality of life

Ind2: spiritual needs $\rightarrow$ Perceived social support $\rightarrow$ spiritual well-being $\rightarrow$ quality of life Ind3: spiritual needs $\rightarrow$ spiritual well-being $\rightarrow$ quality of life

SE: standard error; LLCl: lower limit confidence interval; ULCL: upper limit confidence limit interval.

Figures 


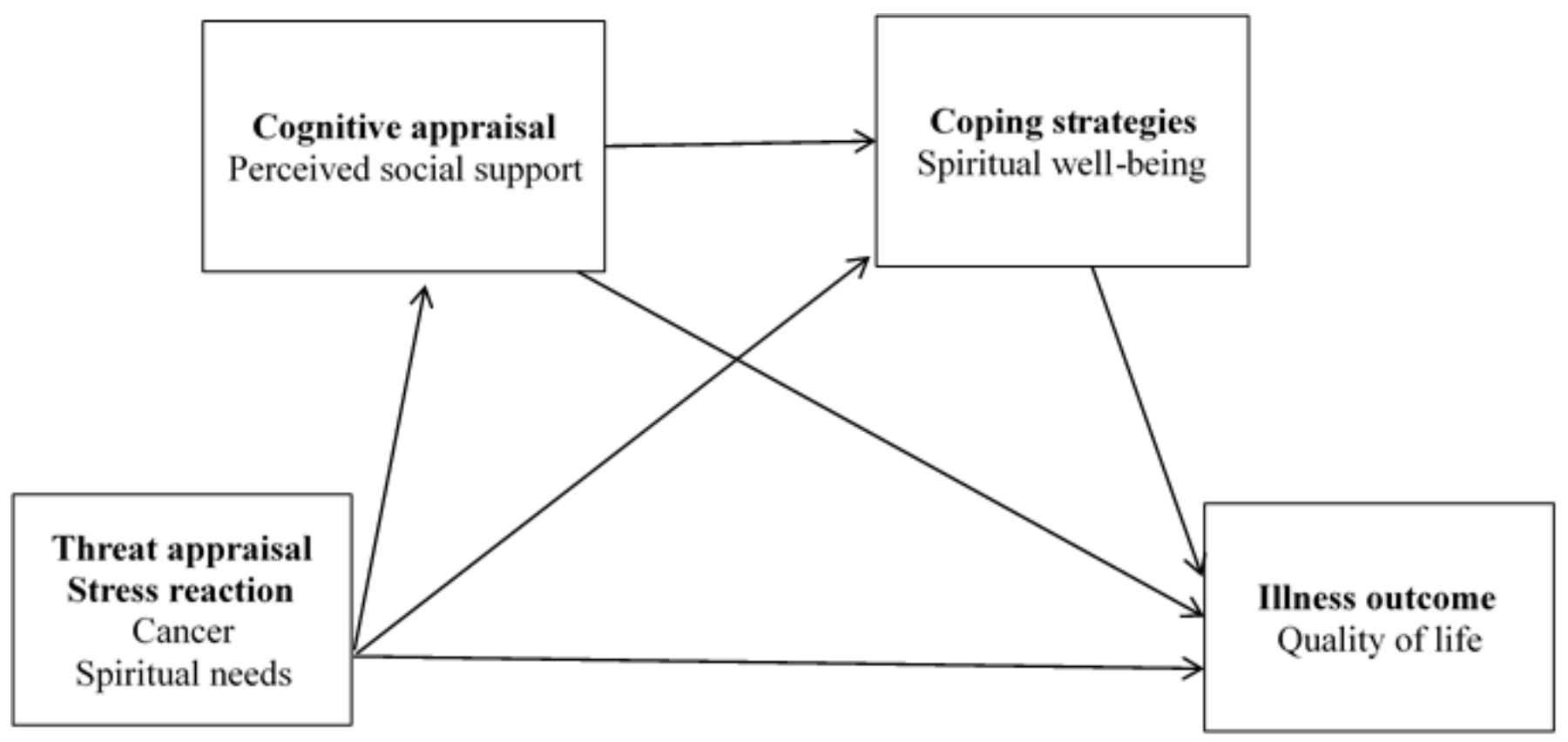

\section{Figure 1}

Proposed serial multiple mediating model of the relationship between Spiritual needs and quality of life based on the Cognitive Appraisal Theory (CAT)

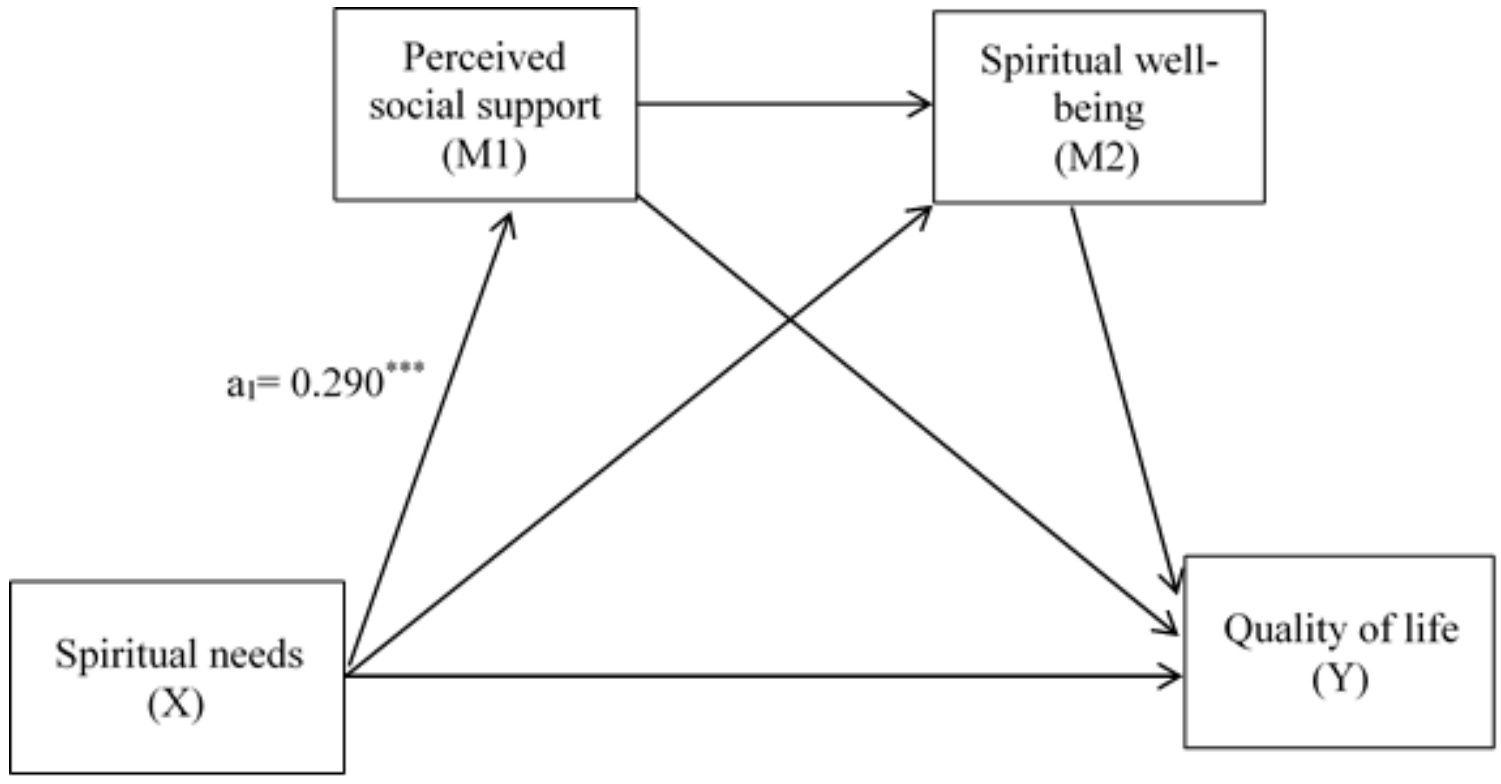

Figure 2

Multiple mediation model showing the direct effect and path coefficients linking spiritual needs to quality of life through illness perceptions and Spiritual well-being in serial $(N=286)$. The coefficient $c$ is the total effect between $X$ and $Y$, and $c^{\prime}$ is the direct effect of $X$ on $Y$ while controlling for the $M 1$ and $M 2$ values in the model are unstandardized regression coefficients. *** $p<0.001 ; ※ \mathrm{P} \otimes 0.05$ 